\title{
Primary diffuse hepatocellular carcinoma in a captive polar bear (Ursus maritimus)
}

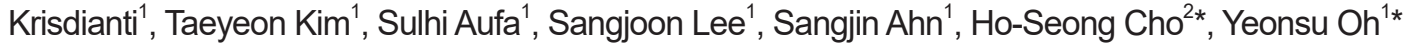 \\ ${ }^{1}$ College of Veterinary Medicine and Institute of Veterinary Science, Kangwon National University, Chuncheon 24341, Korea \\ ${ }^{2}$ College of Veterinary Medicine and Bio-Safety Research Institute, Jeonbuk National University, Iksan 54596, Korea
}

Received November 29, 2021

Revised December 21, 2021

Accepted December 21, 2021

Corresponding author:

Yeonsu Oh

E-mail: yeonoh@kangwon.ac.kr https://orcid.org/0000-0001-5743-5396

Corresponding author:

Ho-Seong Cho

E-mail: hscho@jbnu.ac.kr

https://orcid.org/0000-0001-7443-167X
A captive male polar bear (Ursus maritimus) was found dead after a long-term anorexia at a Park Zoo in Republic of Korea. At necropsy, the liver lesion was prominent with hepatomegaly carrying a big mass and numerous small nodules in various sizes. The cut surface of the nodules was creamy white, firm, and solid with umbilicated appearance. Histologically, there was proliferation of hepatocellular neoplastic cells arranged in glandular-like (pseudoglandular) pattern and complete loss of hepatocellular arrangement. The immunoreactivity for neoplastic hepatocyte antigens was positive for hepatocyte paraffin 1, and negative for vimentin, cytokeratin 7 and 19, and carcinoembryonic antigen. Based on those diagnostic features, the neoplasia was diagnosed as hepatocellular carcinoma. To the author's knowledge, this is the first case report of hepatocellular carcinoma in the captive polar bear in Republic of Korea.

Key Words: Hepatocellular carcinoma, Immunohistochemistry, Polar bear

\section{INTRODUCTION}

Primary hepatic neoplasms are usually composed of hepatocytes, biliary epithelial cells, endothelial cells, or a combination of these with various mesenchymal cells, and typically arise in older animals (Chege et al, 2015). The primary hepatic neoplasms are generally classified as cholangiocarcinoma (CC), hepatocellular adenoma, and hepatocellular carcinoma (HCC) (Goodman, 2007). The CC is a primary adenocarcinoma arising from biliary tracts and considered as the second most frequent primary hepatic neoplasm. The hepatocellular adenoma, a benign tumor, is uncommon but appears solid, and presumable epithelial origin that usually develops in a healthy liver. Meanwhile, the HCC is the most frequent form of primary hepatic neoplasm (Goodman, 2007). It is commonly composed of tumor cells that resemble hepatocytes, and consists of a single variable mass that develops and replaces the parenchyma (Chege et al, 2015). The histological diagnosis of the HCC poses many challenges particularly due to its heterogeneity, and the difficulty to confirm a differential diagnosis in some instances (Quaglia, 2018).

Hepatic neoplasms have been frequently reported in captive wild animals, including Polar bear (Ursus maritimus), Malayan sun bear (U. malayanus), Sloth bear ( $U$. ursinus), Eurasian badger (Meles meles), and antelopes, as a form of HCCs (Hellman et al, 1991; Pettan-Brewer and Lowenstine, 1999; Wilson et al, 2007; Matsuda et al, 2010; Salguero et al, 2010). In domestic animals, hepatic neoplasms were mostly described in dogs, but other animals including cattle, cats, sheep, pigs and horses have also been reported (Jeong et al, 2005; Cullen and Popp, 2016; Mathewos, 2021).

The gross morphology of HCCs are described as massive, nodular, or sometimes diffuse. Massive HCCs are usually presented as a single neoplasm that involves one or contiguous liver lobes, and nodular HCCs forms 
scattered nodules, often within multiple liver lobes. In contrast, diffuse HCCs are characterized by indistinct masses spread throughout the liver parenchyma (Cullen and Pop, 2016).

Histologically, HCCs are characterized by pleomorphic hepatocytes that can form trabeculae, pseudoglandular (acinar), or solid sheets of cells (Cullen and Pop, 2016). The small pseudoglandular pattern may resemble "cholestatic rosettes". Meanwhile, the large pattern is composed of dilated acinar formation with layer of tumor cells as well as fluid content (Matsuda et al, 2010). Mostly, classic HCCs grow in a trabecular pattern, rather pseudoglandular growth is rare (Schlageter et al, 2014).

\section{CASE}

A captive male polar bear, which was raised in a free-range fenced housing to facilitate monitoring, was found dead after a long-term anorexia at a Park Zoo in Republic of Korea. At necropsy, the prominent lesion in the abdominal cavity was shown as the excess accumulation of serosanguinous fluid, implying ascites. Subcutaneous fat and fat around renal capsule were significantly reduced, indicating cachexia which might occurred by several factors including tumor cells behaving as metabolic parasites to promote their growth and survival. These cells lead to excessive ATP production as a result of increased anaerobic metabolism, which converts the glucose to lactate 10 times higher than normal cells. As a consequence, this negative energy balance causes progressive weight loss due to a wasteful form of energy production (Martinez-Outschoorn et al, 2011). Hepatomegaly carrying a big mass, $26 \times 16 \times 12$ $\mathrm{cm}$ in size, and empty gall bladder was observed (Fig. 1). Multiple small nodules of various sizes were dispersed in the liver parenchyma. The cut surface was revealed to be creamy white in firm consistency.

Tissue samples from tumor nodules and representative tissue specimens were collected, fixed in $10 \%(\mathrm{w} / \mathrm{v})$ neutral buffered formalin, routinely processed, and embedded in paraffin. The serial sections at $3 \sim 5 \mu \mathrm{m}$ were used for haematoxylin and eosin (H\&E) staining and immunohistochemistry (IHC). Immunohistochemical (IHC) analyses were performed as previously described (Oh et al, 2016), using six primary antibodies: anti-hepatocyte paraffin-1 (Hep Par 1, Dakocytomation, Glostrup, Denmark), anti-cytokeratin 7 (CK7, Dako), anti-cytokeratin 19 (CK19, Dako), anti-vimentin (Santa Cruz Biotechnology, CA, USA), anti-alpha-fetoprotein (AFP, Dako), and anti-carcinoembryonic antigen (CEA, Dako). Briefly, sections were deparaffinized with xylene and dehydrated through gradient ethanol into water. Antigen retrieval was achieved by proteinase K (Invitrogen, MA, USA) for $25 \mathrm{~min}$ at $37^{\circ} \mathrm{C}$. The endogenous peroxidase was blocked by quenching in $0.3 \%$ hydrogen peroxide solution for 10 min before incubation with primary antibody at $4{ }^{\circ} \mathrm{C}$ overnight, followed by biotinylated secondary antibody for $30 \mathrm{~min}$ at room temperature. Then,

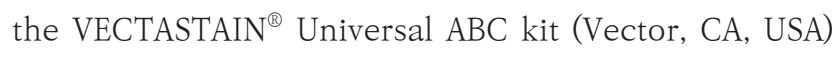
was employed for 30 min. Peroxidase activity was elicited by 3,3'-diaminobenzidine (DAB, Vector, CA, USA), and counterstained with Mayer's hematoxylin (Dako). The negative control was applied by using normal serum instead of primary antibody. Each antibody-treated slide had a corresponding mock-treated slide.

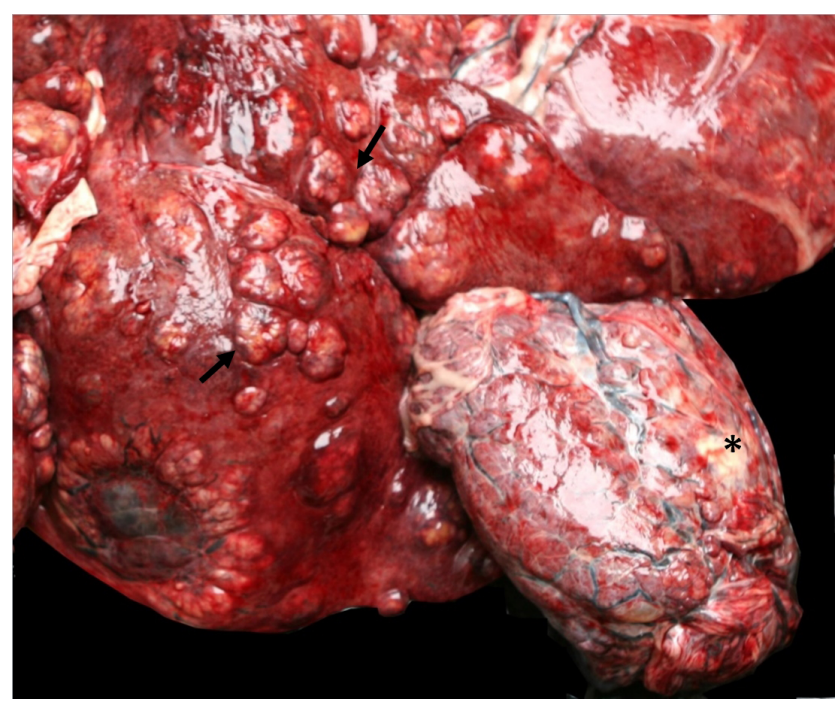

Fig. 1. Liver. Polar bear. Abnormal enlargement carrying a big mass (asterisk) with diffuse multiple nodules in varying sizes (arrows) on the liver. 
Upon histological examination, nodules in liver were composed of acini lined by the proliferation of neoplastic hepatocyte with round to oval nuclei. Very few mitotic figures were viewed (Fig. 2A). The acini are formed by dilatation of bile canalicular-like structures developed by tumor cells and often contain proteinaceous material. The acinar pattern was shown moderate nuclear pleomorphism and prominent endothelial wrapping around tumor cell nests (Fig. 2B). The histomorphological characteristics of the tumor were highly reflective of HCC with pseudoglandular pattern as previously described (Head et al, 2003; Heier et al, 2003; Matsuda et al, 2010).

Hep Par 1 is a component of hepatocellular mitochondria and is expressed in normal and neoplastic hepatocytes with diffuse cytoplasmic granular staining patterns (Matsuda et al, 2010). Hep Par 1 was detected in approximately $90 \%$ of HCC cases in humans, which makes it a suitable marker for this neoplasm (Goodman, 2007). Also, this marker has a sensitivity to reacting with neoplastic and non-neoplastic hepatocytes in polar and brown bears (Heier et al, 2003; Matsuda et al, 2010). Additionally, AFP is a fetal-specific glycoprotein that is normally produced by the fetal yolk sac, liver, and gastrointestinal tract (Zhang et al, 2020). In human, AFP levels is repressed during adult life. However, this glycoprotein is reactivated in more than $70 \%$ of HCC cases and is considered as the most valuable marker for this tumor (Zhou et al, 2006). This specific marker is also observed in brown bear (Matsuda et al, 2010). Nevertheless, some reports described the insensitivity of this protein in canine and human HCC (Chu et al, 2002). In this case, the tumor cells were positive for Hep Par 1 (Fig. 3) in both cytoplasm and nucleus.

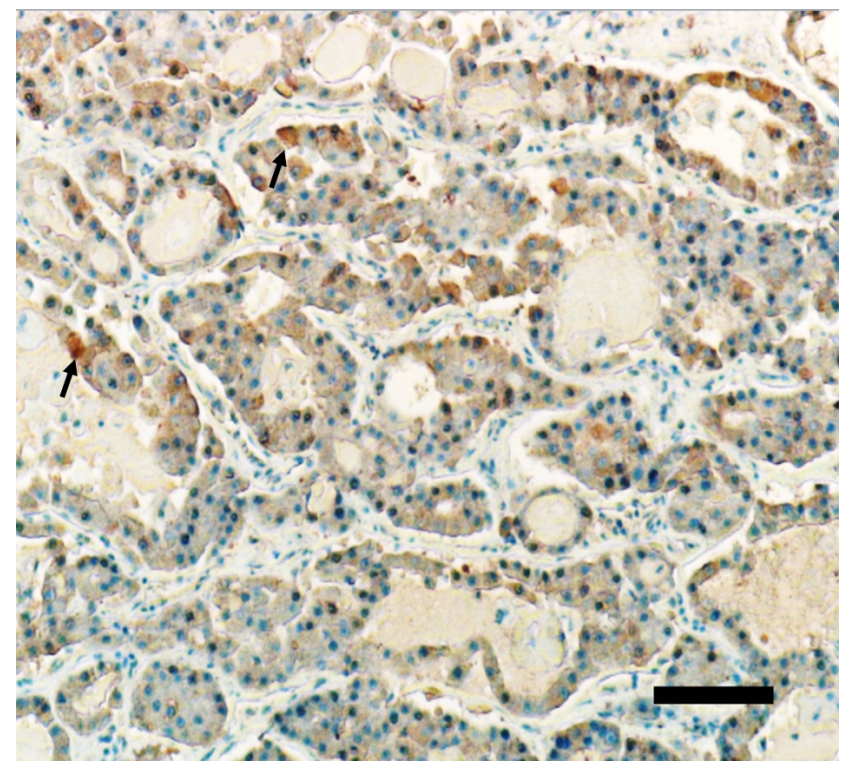

Fig. 3. Liver. Polar bear. Nuclear and cytoplasmic immunoreactivity for hepatocyte paraffin 1 (Hep Par 1). DAB chromogen, hematoxylin counterstain. Scale bar $=100 \mu \mathrm{m}$.
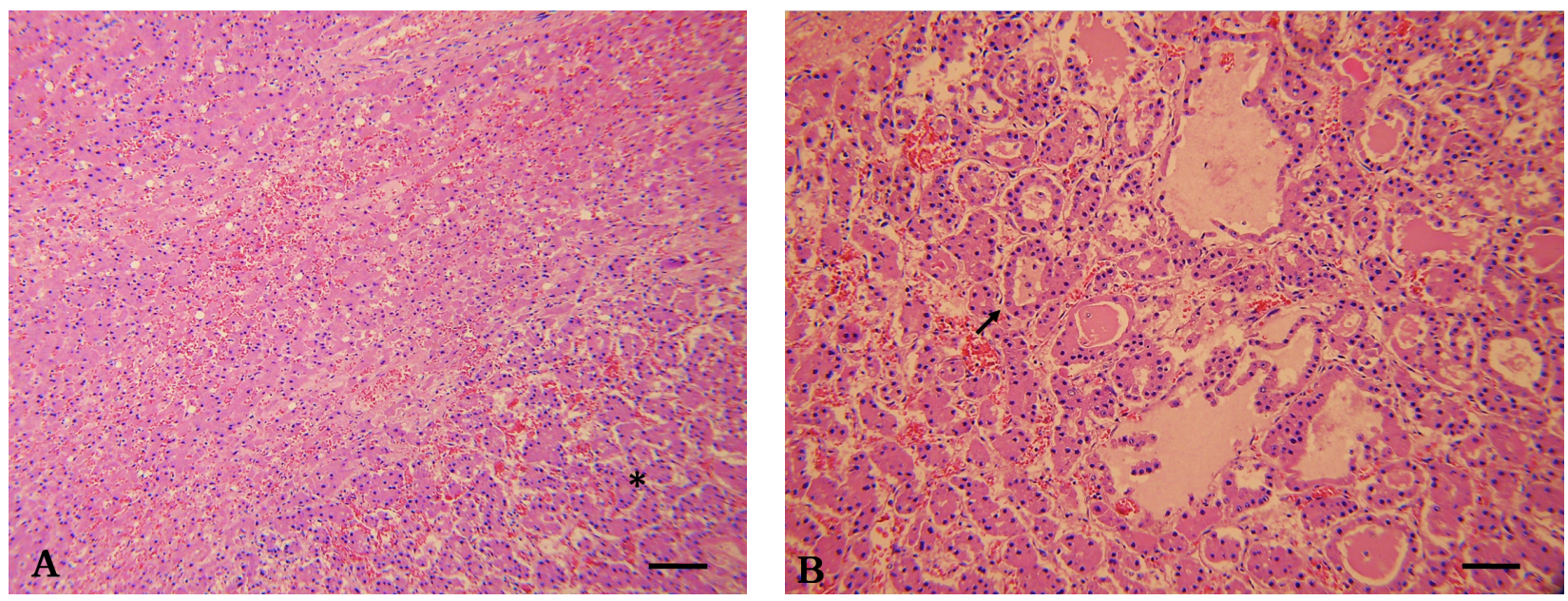

Fig. 2. Liver. Polar bear. (A) Acini (gland-like spaces) lined by the proliferation of hepatocyte neoplastic cells with round to oval nuclei. Very few mitotic figures were viewed. (B) The acini are formed by dilatation of bile canalicular-like structures developed by the tumor cells and often contain proteinaceous material. H\&E stain. Scale bar $=200 \mu \mathrm{m}$. 


\section{DISCUSSION}

To the authors' knowledge, this is the first report of HCC in a captive polar bear in Republic of Korea despite the cause of neoplasm remains unknown. Various etiologic factors for hepatic neoplasms in animals have been discussed. Parasites such as Clonorchis and Opisthrochis flukes, and diets contaminated with heavy and prolonged exposure to hepatic carcinogen, aflatoxin, may cause animal hepatic tumors (Matsuda et al, 2010). Moreover, a genetic component has been proposed to explain the high incidence of liver neoplasms in bears (Petan-Brewwer and Lowenstine, 1999). Additionally, hepatic stellate cells in polar bears are perceived to have comparatively high vitamin Astoring capacity. Metabolites of vitamin A are known to profoundly effect a wide variety of biological actions including cell proliferation, differentiation, morphogenesis, and tumorigenesis (Senoo et al, 2012). Vitamin Arelated hepatic carcinogenesis was reported in human case, as well as in polar bears (Heier et al, 2003). Hence, an imbalance of vitamin A in polar bears' diet will most likely give rise to health issues for the animals. In conclusions, an elderly polar bear in captivity was found dead at a zoo, and the cause of death was determined to be HCC. Although it is difficult to elucidate the etiology of senile malignant tumors, the relationship with vitamin A, which has been mentioned in the literature, cannot be excluded.

\section{ACKNOWLEDGEMENTS}

This work was supported by Korea Institute of Planning and Evaluation for Technology in Food, Agriculture and Forestry (IPET) through Livestock Industrialization Technology Development Program, funded by Ministry of Agriculture, Food and Rural Affairs (MAFRA)(No. 321081-4).

\section{CONFLICT OF INTEREST}

No potential conflict of interest relevant to this article was reported.

\section{ORCID}

Krisdianti, https://orcid.org/0000-0003-4062-7484

Taeyeon Kim, https://orcid.org/0000-0003-3345-9478

Sulhi Aufa, https://orcid.org/0000-0003-4951-0231

Sangjoon Lee, https://orcid.org/0000-0002-2498-8397

Sangjin Ahn, https://orcid.org/0000-0003-1821-6101

Ho-Seong Cho, https://orcid.org/0000-0001-7443-167X

Yeonsu Oh, https://orcid.org/0000-0001-5743-5396

\section{REFERENCES}

Chege S, Vissiennon T, Cavero T, Kinne J, Toosy A. 2015. A rare case of hepatocellular carcinoma in the arabian sand cat (Felis margarita harrisoni). J Zoo Wild Med. 46: 699-703.

Chu PG, Ishizawa S, Wu E, Weiss LM. 2002. Hepatocyte antigen as a marker of hepatocellular carcinoma: an immunohistiochemical comparison to carcinoembryonic antigen, CD10, and alpha-fetoprotein. Am J Surg Pathol. 26: 978-988.

Cullen JM, Popp JA. 2016. Tumors of the liver and gall bladder. In Meuten DJ (Ed.), Tumors in Domestic Animals. $5^{\text {th }}$ Ed. (pp 486-490). John Wiley and Sons, Hoboken, New Jersey.

Goodman Z. 2007. Neoplasms of the liver. 20, S49-S60. Head KW, Cullen JM, Dubielzig RR, Else RW, Misdorp W, Patnaik AK, Tateyama S, van der Gaag I. 2003. In Schulman FY (Ed.), Histological Classification of Tumors of the Alimentary System of Domestic Animals. (pp. 119-135). Armed Forces Institute of Pathology, Washington DC.

Heier A, Gröne A, VöüVöllm J, Kübber-Heiss A, Bacciarini LN. 2003. Immunohistochemical study of retinol-binding protein in livers of polar bears (Thalarctos maritimus). Vet Pathol. 40: 196-202. 
Hellmann J, Hofmeister R, Göltenboth R. 1991. The occurrence of tumors in large bears (Ursidae)--a literature review and six case descriptions. Berl Munch Tierarztl Wochenschr. 104: 262-268.

Jeong WI, Do SH, Sohn MH, Yun HS, Kwon OD, Kim TH, Jeong DH, Williams BH, Jeong KS. 2005. Hepatocellular carcinoma with metastasis to the spleen in a Holstein cow. Vet Pathol. 42: 230-232.

Martinez-Outschoorn UE, Pestel RG, Howell A, Tykocinski ML, Nagajyothi F, Machado F, Tanowitz HB, Sotgia F, Lisanti MP. 2011. Energy transfer in "parasitic" cancer metabolism. Cell cycle. 10: 42084216.

Mathewos M. 2021. Pathological and Cytological Studies on Hepatocellular Carcinoma in Cattle Slaughtered at Bishoftu Elfora Abattoir, Central Ethiopia. Vet Med Int. 2021: 6649172.

Matsuda K, Qiu Y, Kawamura Y, Suzuki H, Takita Y, Sakamoto H, Sasaki K, Taniyama H. 2010. Hepatocellular carcinoma in a Hokkaido brown bear (Ursus arctos yesoensis). J Vet Med Sci. 72: 1213-1216.

Oh Y, Kim S, Oh S, Han J. 2016. Primary diffuse malignant peritoneal mesothelioma in a striped skunk (Mephitis mephitis). J Vet Med Sci. 78: 485-487.

Pettan-Brewer KC, Lowenstine LJ. 1999. Intrahepatic cysts and hepatic neoplasms in felids, ursids, and other zoo and wild animals. pp. 426-427. In: Fowler ME, Miller RE (Eds.), Zoo \& Wild Animal Medi- cine. Current Therapy, 4th ed. W. B. Saunders Co., Philadelphia, Pennsylvania.

Quaglia A. 2018. Hepatocellular carcinoma: a review of diagnostic challenges for the pathologist. J Hepatocell Carcinoma. 5: 99-108.

Salguero FJ, Richard A, Gough J, Long A, Weyer U, Cooley WA, Chambers MA, Lesellier S. 2010. Pelioid hepatocellular carcinoma in an adult Eurasian badger (Meles meles). J Comp Pathol. 142: 208-212.

Schlageter M, Terracciano LM, D’Angelo S, Sorrentino P. 2014. Histopathology of hepatocellular carcinoma. World J Gastroenterol. 20: 15955-15964.

Senoo H, Imai K, Mezaki Y, Miura M, Morii M, Fujiwara M, Blomhoff R. 2012. Accumulation of vitamin A in the hepatic stellate cell of arctic top predators. Anat Rec. 295: 1660-1668.

Wilson FD, Fitzgerald SD, Kiupel M, Walker RL, Williams CB, Todd DJ. 2007. Occurrence of hepatocellular carcinoma in an adult male Nile lechwe (Kobus megaceros). J Zoo Wildl Med. 38: 329-332.

Zhang J, Chen G, Zhang P, Zhang J, Li X, Gan D, Cao X, Han M, Du H, Ye Y. 2020. The threshold of alphafetopotein (AFP) for the diagnosis of hepatocellular carcinoma: A systematic review and metanalysis. PLoS ONE. 15: e0228857.

Zhou L, Liu J, Luo F. 2006. Serum tumor markers for detection of hepatocellular carcinoma. World J Gastroenterol. 12: 1175-1181. 